\title{
Early pregnancy termination with a simplified mifepristone: Medical abortion outpatient regimen
}

\section{Chuni $\mathbf{N}^{1}$, Chandrashekhar TS ${ }^{2}$}

${ }^{1}$ Associate professor, Department of Obstetrics \& Gynaecology, ${ }^{2}$ Lecturer, Department of Community Medicine, Manipal College of Medical Sciences, Pokhara, Nepal.

\begin{abstract}
Background: An estimated 30 million abortions are performed worldwide every year. Many women do not have access to abortion and die of complications after illegal abortions. Medical abortion could provide greater access to safe abortion services; availability of the procedure is, therefore, of global public health importance.

Aim: The aim was to study the efficacy of lowered dose of Mifepristone in medical abortion.

Materials and methods: One hundred and twelve cases with a pregnancy of 63 days duration or less were enrolled in a prospective study using a lowered dose of 200mg Mifepristone followed, 48 hours later, by home administration of $400 \mu \mathrm{g}$ Misoprostol orally. At the second visit, on day 15, outcome and adverse effects were analysed. Women who failed to undergo a complete abortion were further managed by surgical evacuation of uterus.

Results: The mean gestational age was 50.6 days. The rates of complete abortion were $92.8 \%, 83 \%$ and $80 \%$ in the $\leq 49$ days group, 50 to 56 days and 57 to 63 days group respectively. Vaginal bleeding emerged as the biggest reason for medically indicated termination. Nulliparous women had a greater frequency of side effects, though values did not reach statistical significance.

Conclusion: This regimen of a lower dose of 200mg Mifepristone, followed by home administration of $400 \mu \mathrm{g}$ oral Misoprostol 48 hours later is safe and highly effective especially in pregnancies of up to 49 days duration.
\end{abstract}

Key words: Mifepristone, Medical termination of pregnancy (MTP)

\begin{abstract}
A $\mathrm{n}$ estimated 30 million abortions are performed worldwide every year. Many women do not have access to abortion and in developing countries up to 200,000 women die annually of complications after illegal abortions ${ }^{1}$. Medical abortion could provide greater access to safe abortion services; availability of the procedure is, therefore, of global public health importance.
\end{abstract}

The traditionally approved regimen consists of oral administration of $600 \mathrm{mg}$ Mifepristone followed in 48 hours by oral administration of $400 \mu \mathrm{g}$ Misoprostol, with both drugs taken in the provider's clinic and a follow up visit around day 14. A growing amount of literature has shown a comparable level of efficacy with a lower dose of $200 \mathrm{mg}$ Mifepristone, combined with varying routes and doses of Misoprostol.

This article reports a prospective trial of $200 \mathrm{mg}$ Mifepristone followed 48 hours later by home administration, of asingle oral dose of $400 \mu \mathrm{g}$ Misoprostol. The aim of this study was to produce information in two aspects; namely, lowering the dose of Mifepristone to $200 \mathrm{mg}$ and incorporating administration of Misoprostol at home. Both these measures were aimed at reducing cost and number of clinical visits.

\section{Materials and methods}

The study was conducted at Sikkim Manipal Institute of Medical Sciences, Gangtok, from January 2004 to January 2005. One hundred and twelve women, each with a documented pregnancy of 63 days duration or less, requesting termination of pregnancy medically, were enrolled in the study. All the women were between 18 to 35 years of age. Women with signs and symptoms of threatened abortion, ectopic pregnancy, renal, liver, respiratory, cardiovascular or adrenal disease, hypertension, diabetes, anaemia, undiagnosed adnexal mass, coagulopathy or known allergy to prostaglandins were excluded. Smokers and women who were breastfeeding, or receiving long term glucocorticoid or

\footnotetext{
Correspondence

Dr. Neena chuni

Department of OBS \& Gynae,

Manipal Teaching Hospital,

Phulbari, Pokhara, Nepal

E-mail: nchuni@gmail.com
} 
anticoagulant therapy were also excluded. All women agreed to undergo surgical termination of pregnancy if the medical method failed.

\section{Study Design}

A total of 112 patients enrolled into the study and 107 women returned for the second visit on day 15 . At the initial visit, on day 1 , counselling was done and all women gave written and informed consent. A complete medical history, physical examination and gestational age estimation were performed. Gestational age was assessed on the basis of menstrual history, bimanual examination and transvaginal ultrasonography. On the basis of these three measures, women were assigned to the following arbitrarily defined gestational age groups: the $\leq 49$ days group (42 women); 50 to 56 days group ( 35 women); and 57 to 63 days group ( 30 women). Women who met the enrolment criteria and gave written informed consent ingested 200mg Mifepristone orally. Each woman was given two tablets of $200 \mu \mathrm{g}$ Misoprostol to take home and instructed to take those tablets orally in 48 hours. They were also given medication for pain. These women were also given a card on which to record adverse events such as nausea, vomiting, diarrhoea and abdominal pain. Vaginal bleeding was recorded by each woman on days 1 to 15 as spotting (less than normal menstrual bleeding), normal (similar to normal menstrual bleeding), or heavy (more than normal menstrual flow). Patients also received information describing symptoms requiring emergency treatment (such as very heavy bleeding, fever and severe pain). At the second visit on day 15, outcome of the medical abortion regimen was classified into one of four categories:

1. Complete abortion (requiring no treatment);

2. Incomplete abortion (products of conception passed but clinical or ultrasound signs of incomplete abortion);

3. Missed abortion (no products passed and ultrasound evidence of retained gestation sac but no cardiac activity); and

4. Continuing pregnancy (no products passed and cardiac activity present on ultrasound).

Women with treatment outcomes in the last three categories were further managed by surgical evacuation of the uterus. Follow up was extended beyond second visit only if bleeding persisted. All statistical analysis was carried out by using commercially available software (Statistical Package for the Social Sciences, SPSS Inc.) and statistical significance was defined as having a $\mathrm{p}$ value. 05 .
Results

During the study period, 112 women enrolled in the study. Five women failed to return for the second visit on day 15 and were excluded from efficacy analysis with lack of evidence of either failure or success. Thus, 107 women remained in the final analysis.

The mean age of the study participants was 28 years. 102 (95\%) women had previous pregnancies; and more than half $(60.7 \%)$ had a previous abortion. Majority of patients $(39.2 \%)$, had a period of gestation between 43 and 49 days. The mean gestational age was 50.6 days. The three gestational groups were similar in respect to age, gravidity, parity, and number of previous abortions.

Among the 107 women who returned for the second visit, the rates of pregnancy termination were $92.8 \%$ in the $\leq 49$ days group, $83 \%$ in the 50 to 56 days group and $80 \%$ in the 57 to 63 days group. These differences were not statistically significant. $(\chi 2 \mathrm{p}$ value $>0.05)$. The rates of incomplete abortion were $8.6 \%$ in the 50 to 56 days group and $10 \%$ in the 57 to 63 days group, as compared with $4.8 \%$ in the $\leq 49$ days group.(Table 1). Ongoing pregnancy was seen in only 2 women $(1.8 \%)$, both in the 57 to 63 days group. Vaginal bleeding is an expected and natural consequence of the abortion process. The mean duration of heavy bleeding (bleeding heavier than menses) was 2 days, bleeding similar to menses was 5.2 days and spotting 3.8 days. Vaginal bleeding was the most common reason for surgical intervention $(80 \%)$. Blood transfusion was, however, not required in any patient. Patient's request was the reason least often cited for surgical intervention (1\%).

The most common adverse effects reported by patients were pain, nausea, vomiting, diarrhoea, headache and weakness (Table2). Almost all the women reported at least one adverse event. Nearly all the women in the three groups had abdominal pain. Abdominal pain not relieved by analgesics was the reason for one medically indicated surgical evacuation. As compared with the $\leq 49$ days group, the other two groups had higher rates of nausea; and vomiting was significantly more in these two groups $(\chi 2 \mathrm{p}$ value $<0.05)$. Other adverse events reported included back pain $(36 \%)$, dizziness $(12 \%)$, fever $(4 \%)$, anorexia $(3 \%)$, syncope $(2 \%)$ and vaginal discharge $(2 \%)$. It was seen that nulliparous women had a greater frequency of side effects, though values did not reach statistical significance. 
Table 1: Treatment outcome and indications for surgical evacuation according to gestation

\begin{tabular}{|c|c|c|c|c|}
\hline \multirow{2}{*}{ Outcome } & \multicolumn{3}{|c|}{ Period of gestation } & \multirow{2}{*}{ Total } \\
\hline & $\leq 49$ days $(n=42)$ & 50-56days $(n=35)$ & 57-63days $(n=30)$ & \\
\hline Success & $32(92.8)$ & $29(83)$ & $24(80)$ & $92(86)$ \\
\hline Failure & $3(7.1)$ & $6(17.1)$ & $6(20)$ & $15(14)$ \\
\hline Medical indication & $1(2.3)$ & $2(5.7)$ & $2(6.7)$ & $5(4.6)$ \\
\hline Patients request & - & $1(2.8)$ & - & $1(1)$ \\
\hline Incomplete abortion & $2(4.8)$ & $3(8.6)$ & $3(10)$ & $8(7.7)$ \\
\hline Ongoing pregnancy & - & - & $2(6.6)$ & $2(1.8)$ \\
\hline
\end{tabular}

Figures in parenthesis are percentages

Table 2: Incidence of adverse effects according to gestational age

\begin{tabular}{|l|c|c|c|}
\hline \multirow{2}{*}{ Adverse effect } & \multicolumn{3}{|c|}{ Period of gestation } \\
\cline { 2 - 4 } & <49days $(\mathbf{n}=\mathbf{4 2})$ & $\mathbf{5 0 - 5 6 d a y s ~ ( n = 3 5 )}$ & $\mathbf{5 7 - 6 3 d a y s ~ ( n = 3 0 )}$ \\
\hline Abdominal pain & $40(95.2)$ & $34(97.1)$ & $29(96.6)$ \\
\hline Nausea & $26(62)$ & $25(71.4)$ & $22(73.3)$ \\
\hline Vomiting & $11(26.1)$ & $13(37.1)$ & $17(40.4)$ \\
\hline Diarrhoea & $8(19.4)$ & $8(40.4)$ & $7(23.3)$ \\
\hline
\end{tabular}

Figures in parenthesis are percentages

\section{Discussion}

The present study tested a regimen of lower dose of Mifepristone from $600 \mathrm{mg}$ to $200 \mathrm{mg}$ and patient self administration at home of the $400 \mu \mathrm{g}$ oral Misoprostol. The results confirm that this is a safe and effective regimen for early pregnancy termination. The overall success rates for various gestational ages ranged from $80 \%$ to $92.8 \%$. The success rate seen in the group with amenorrhea, 49 days or less, is not statistically different from that found in the U.S. registration trial of 827 women ${ }^{2}$, though the success rates for amenorrhea greater than 49 days is lesser than $91 \%$ to $96 \%$ reported in other studies $^{3,4,5}$. A decrease in efficacy after 49 days has been reported with Misoprostol but not with Gemeprost ${ }^{6}$.

Thus, the prostaglandin component and not Mifepristone appears to be related to the lower success rates. Lower rates were not found when Misoprostol was given by the vaginal route ${ }^{7}$, presumably because of greater tissue bioavailability ${ }^{8}$.

The incidence of adverse effects rose with the duration of pregnancy, but this was not statistically significant, except in the case of vomiting [Table 2]. Although the aggregate failure rate (14\%) and the rate of incomplete abortion $(7.7 \%)$ is higher than that reported by Spitz et al (5.9\% and $4.7 \%$ respectively) [2], the rate of $1.8 \%$ for ongoing pregnancy was similar( $1 \%)$. The reasons for these differences are not clear, but previous history of elective abortions could have contributed to these differences, because $53 \%$ of the women in our study had had elective abortions previously, as compared with 25 to 27 percent in previous studies [7].

Almost all the patients had bleeding, pain and gastrointestinal complaints but these were mild and resulted in only three hospitalisations. No patient required a blood transfusion. Five patients were lost to follow up. The outcome of these pregnancies is not known. Although there are no human studies available for possible teratogenicity due to Mifepristone, Misoprostol has been reported to be teratogenic in humans ${ }^{9}$. Therefore, careful follow up is essential to ensure surgical termination in cases with failure of medical abortion.

\section{Conclusion}

This regimen of reduced dose of Mifepristone, followed by home administration of $400 \mu \mathrm{g}$ oral Misoprostol 48 hours later is safe and highly effective in pregnancies of up to 49 days duration. This method is particularly recommended in developing countries like Nepal, where trained manpower to perform surgical termination may not be available to all. Reduced costs with a lower dose of Mifepristone and limited number of clinical visits make this method particularly attractive to women and ensure more privacy in those countries where induced abortions are still not socially acceptable. With the legalisation of abortions in Nepal, these drugs should be registered and introduced in the country, along with provider training for familiarity with medical abortion. 


\section{References}

1. Henshaw SK. Induced abortion: a world review, 1990. Fam Plann Perspect. 1990;22:76-89. [Erratum, Fam Plann Perspect 1990;22:114]

2. Spitz IM, Bardin CW, Benton L, Robbins A. Early pregnancy termination with mifepristone and misoprostol in the United States. N Engl J Med. 1998;338:1241-7.

3. World Health Organization Task force on Postovulatory Methods of Fertility Regulation. Comparison of two doses of mifepristone in combination with misoprostol for early medical abortion: a randomized trial. BJOG. 2000; 107:524-30

4. Elul B, Hajri S, Ngoc NN, Ellertson C, Slama $\mathrm{CB}$, Pearlman E, et al. Can women in developing countries use a simplified medical abortion regimen? Lancet. 2001; 357: 1402-5.

5. Hajri S, Blum J, Gueddana N, Saadi H, Maazoun L, Chelli H, et al: Expanding medical abortion in Tunisia: women's experiences from a multi-site expansion study. Contraception. 2004;70:487-91.
6. The efficacy and tolerance of mifepristone and prostaglandin in termination of pregnancy of less than 63 days gestation: U K Multicenter Studyfinal results. Contraception. 1997; 55:1-5.

7. Schaff EA, Stadalius LS, Eisinger SH, Franks P. Vaginal misoprostol administered at home after mifepristone (RU 486) for abortion. J Fam Pract. 1997; 44:353-60.

8. Zieman M, Fong SK, Benowitz NL, Banskter D, Darney PD. Absorption kinetics of misoprostol with oral or vaginal administration. Obstet gynecol. 1997; 90: 88-92.

9. Gonzalez $\mathrm{CH}$, Vargas $\mathrm{FR}$, Perez $\mathrm{AB}$, et al. Limb deficiency with or without Mobius sequence in seven Brazilian children associated with misoprostol use in the first trimester of pregnancy. Am J Med Genet. 1993;47:59-64. 